\title{
LSD impairs working memory, executive functions, and cognitive flexibility, but not risk-based decision making
}

Thomas Pokorny, MSc ${ }^{1,2 *}$, Patricia Duerler, MSc ${ }^{1,2}$, Erich Seifritz, MD $^{3}$, Franz X.

\author{
Vollenweider, $\mathrm{MD}^{1,2}$, Katrin H. Preller, $\mathbf{P h D}^{1,2}$
}

\begin{abstract}
${ }^{1}$ Neuropsychopharmacology and Brain Imaging, Department of Psychiatry, Psychotherapy and Psychosomatics, Psychiatric Hospital, University of Zurich, Zurich, Switzerland ${ }^{2}$ Heffter Research Center Zurich, Department of Psychiatry, Psychotherapy and Psychosomatics, Psychiatric Hospital, University of Zurich, Zurich, Switzerland

${ }^{3}$ Department of Psychiatry, Psychotherapy and Psychosomatics, Psychiatric Hospital, University of Zurich, Zurich, Switzerland
\end{abstract}

\section{Abbreviated Running Title}

LSD impairs cognition via 5-HT2A receptor activation

\section{*Correspondence to:}

Thomas Pokorny

Neuropsychopharmacology and Brain Imaging

and Heffter Research Center Zurich

Department of Psychiatry, Psychotherapy and Psychosomatics

University Hospital of Psychiatry Zurich

Lenggstrasse 31

CH-8032 Zurich

Switzerland

Phone +41 443842603

Fax +41 443842499

e-mail: thomas.pokorny@usz.ch 
Pokorny et al.

\begin{abstract}
Psychiatric and neurodegenerative illnesses are characterized by cognitive impairments, in particular deficits in working memory, decision making, and executive functions including cognitive flexibility. However, the neuropharmacology of these cognitive functions is poorly understood. The serotonin (5-HT) 2A receptor might be a promising candidate for the modulation of cognitive processes. However, pharmacological studies investigating the role of this receptor system in humans are rare. Recent evidence demonstrates that the effects of Lysergic acid diethylamide (LSD) are mediated via agonistic action at the 5- $\mathrm{HT}_{2 \mathrm{~A}}$ receptor. Yet, the effects of LSD on specific cognitive domains using standardized neuropsychological

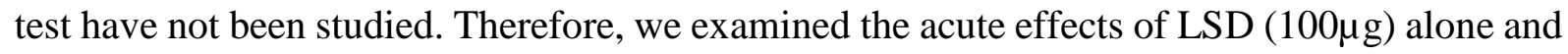
in combination with the 5- $\mathrm{HT}_{2 \mathrm{~A}}$ antagonist ketanserin (40mg) on cognition, employing a double-blind, randomized, placebo-controlled, within-subject design in 25 healthy participants. Executive functions, cognitive flexibility, spatial working memory, and risk-based decisionmaking were examined by the Intra/Extra-Dimensional shift task (IED), Spatial Working Memory task (SWM), and Cambridge Gambling Task (CGT) of the Cambridge Neuropsychological Test Automated Battery. Compared to placebo, LSD significantly impaired executive functions, cognitive flexibility, and working memory on the IED and SWM, but did not influence quality of decision-making and risk taking on the CGT. Pretreatment with the 5- $\mathrm{HT}_{2 \mathrm{~A}}$ antagonist ketanserin normalized all LSD-induced cognitive deficits. The present findings highlight the role of the 5- $\mathrm{HT}_{2 \mathrm{~A}}$ receptor system in executive functions and working memory and suggest that specific $5-\mathrm{HT}_{2 \mathrm{~A}}$ antagonists may be relevant for improving cognitive dysfunctions in psychiatric disorders.
\end{abstract}


Pokorny et al.

\section{Introduction}

Most psychiatric and neurodegenerative illnesses are characterized by cognitive impairments (Claesdotter, et al, 2018; Jagust, 2018; Wang, et al, 2019; Wunderli, et al, 2016). These deficits have a deleterious effect on patients' quality of life and are severely impairing real world functioning (Millan, et al, 2012). Previous research has described trans-diagnostic impairments in various cognitive domains in patients (Millan, et al, 2012). In particular, deficits in executive functions, working memory, and decision making are among the most common affected domains in psychiatric disorders and have been observed in highly prevalent illnesses like depression, schizophrenia, and substance use disorders (Jessen, et al, 2018). While some existing pharmacological treatments have been shown to improve cognitive performance, these effects are small (Désaméricq, et al, 2014). Other currently used drugs such as first-generation antipsychotics may even worsen cognitive functions (Hill, et al, 2010). Deficits in cognitive abilities are therefore a highly important trans-diagnostic dimension in psychiatric and neurological disorders with great need for improved treatment (Millan, et al, 2012).

Pharmacological studies offer the opportunity to causally investigate the contribution of specific receptors and therefore elucidate the neuropharmacological basis of cognitive deficits. This knowledge is urgently needed for the development of specific and novel treatment approaches. Abnormal signalling of the serotonin (5-HT) 2A receptor has been reported in various psychiatric disorders (Zhang and Stackman, 2015). Furthermore, this receptor is widely distributed in brain regions important for cognition and learning (Zhang and Stackman, 2015). However, pharmacological studies investigating the role of this receptor system in humans are rare (Zhang and Stackman, 2015).

Lysergic acid diethylamide (LSD) is a classical hallucinogenic compound and has been shown to transiently induce subjective psychedelic experiences as well as alterations in brain activity and connectivity via agonistic activity on the 5- $\mathrm{HT}_{2 \mathrm{~A}}$ receptor (Preller, et al, 2018a; Preller, et al, 2017; Preller, et al, 2018b). The administration of LSD therefore offers the opportunity to causally elucidate the role of the $5-\mathrm{HT}_{2 \mathrm{~A}}$ receptor in human cognition. Two recent studies reported that LSD subjectively decreased concentration and increased the self-report of cognitive disorganization (Carhart-Harris, et al, 2016; Schmid, et al, 2015). Yet, objective measures of cognitive abilities under LSD are still lacking. Therefore, the present study investigated the acute effects of LSD on executive functions, spatial working memory, and riskbased decision-making using computerized and standardized tests provided by the Cambridge Neuropsychological Automated Test Battery (CANTAB). Furthermore, while previous studies 
point to the involvement of the 5- $\mathrm{HT}_{2 \mathrm{~A}}$ receptor in LSD-induced effects (Preller, et al, 2017), the contribution of this receptor subtype to alterations in cognitive processes is unknown. To be able to investigate the specific role of the 5-HT2A receptor in cognition, we blocked this receptor subtype via the pre-treatment of LSD with the 5-HT2A receptor antagonist ketanserin. We hypothesized that 1) LSD impairs executive functions, spatial working memory, and riskbased decision-making and 2) that these alterations are attributable to LSD’s agonistic activity on the $5-\mathrm{HT}_{2 \mathrm{~A}}$ receptor. 
Pokorny et al.

\section{Methods}

\section{Participants}

Twenty-five healthy participants (19 men, 6 women, mean age \pm SD: 25.24 \pm 2.79 , mean verbal IQ \pm SD: 108.4 \pm 9.2 ) were enrolled in the study. All participants underwent a screening procedure at the Department of Psychiatry, Psychotherapy and Psychosomatic, Psychiatric Hospital, University of Zurich consisting of a psychiatric interview (M.I.N.I., (Sheehan, et al, 1998), laboratory test (blood chemistry and urinalysis for drug and pregnancy screening), and a routine medical examination including electrocardiogram. Verbal intelligence was measured with the German version of a multiple choice vocabulary intelligence test (Lehrl, 2005).Volunteers were included when they were 20-40 years of age and willing to refrain from consuming psychoactive drugs at least two weeks before the first experimental session and during the study. The exclusion criteria were personal or first-degree relative history of psychiatric disorders, acute or chronic physical illness, cardiovascular diseases, history of head trauma, neurological diseases such as migraine headaches and epilepsy, history of drug dependence or abuse, a previous significant adverse response to a hallucinogenic drug, and pregnancy or lactation. Before participating, all participants gave their written consent after having received detailed written and oral information about the aims of the study, and the effects and possible risks of the substances administered in accordance with the Declaration of Helsinki. The study was approved by the Ethics Committee of the Department of Public Health of the Canton of Zurich, Switzerland, and the use of LSD was authorized by the Swiss Federal Office for Public Health, Department of Pharmacology and Narcotics, Berne, Switzerland. The current data were collected as part of a larger study (Kraehenmann, et al, 2017; Preller, et al, 2017; Preller, et al, 2018b) and the study was registered at clinicaltrials.gov (NCT02451072).

\section{Study design and experimental procedures}

This study employed a double-blind, randomized, placebo-controlled, within-subject design with three experimental sessions, each separated by at least two weeks. All participants underwent three drug conditions: placebo+placebo (Pla), placebo+LSD (LSD), and ketanserin+LSD (Ket+LSD). One hour after the intake of the first capsule (placebo: 179mg mannitol, 1mg aerosol, p.o.; or ketanserin: 40mg, p.o.) the second one (placebo: 179mg

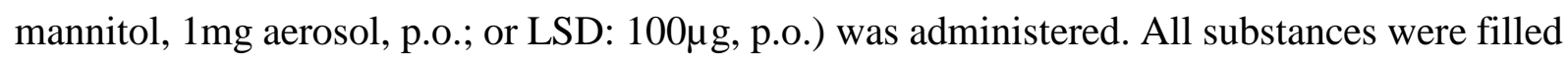
in identical looking gelatine capsules. A urine test for drug-screening and pregnancy-test was conducted at the beginning of each experimental session before drug administration. Participants completed the Intra/Extra-Dimensional shift task (IED), Spatial Working Memory 
task (SWM), and Cambridge Gambling Task (GCT, CANTABeclipse 5.0.12, Cambridge Cognition Ltd., Cambridge, UK) on a computer with a 18" touch-sensitive screen (Elo Touch Solutions) in a quiet room 220 minutes after the administration of the second capsule. Participants completed the Five Dimension Altered State of Consciousness (5D-ASC) questionnaire (Dittrich, 1998) 720 minutes after drug intake to retrospectively rate subjective drug effects.

\section{Questionnaire and cognitive tasks}

\section{Altered States of Consciousness Rating Scale (5D-ASC)}

The 5D-ASC (Dittrich, 1998) was used to assess subjective drug effects in each session. Scores were calculated for eleven validated subscales (Studerus, et al, 2010): experience of unity, spiritual experience, blissful state, insightfulness, disembodiment, impaired control and cognition, anxiety, complex imagery, elementary imagery, audio-visual synesthesia, and changed meaning of percepts. Results of the 5D-ASC data are expressed as percentage scores of maximum absolute subscale values.

\section{Executive Functions: Intra/Extra-Dimensional shift task (IED)}

The IED is a test of rule acquisition and cognitive shifting and represents a computerised analogue of the Wisconsin Card Sorting test. Besides attentional set-shifting the task measures different cognitive abilities such as discriminative learning, reversal learning, formation of an attentional set, shifting of attention within the same dimension (intra-dimensional shift, IDS) and between different perceptual dimensions (extra-dimensional shift, EDS). Perceptual dimensions are operationalized via two stimulus characteristics (purple-filled shapes or white lines). Attentional set-shifting is a measure of cognitive flexibility and executive functions. The task consists of nine learning stages, during which the participant first has to focus on shapes or lines within a relevant dimension (IDS) and then shift attention to a previously irrelevant dimension (EDS, stage 8, for a detailed description see Pantelis, et al, 2009). The measures of performance on this task are: number of stages completed (stages completed), total number of errors made across all stages adjusted for stages not completed (number of errors adjusted) representing a measure of subject's efficiency in attempting the test, the number of errors on individual stages (number of errors), and the mean time to reach a decision within individual stages (total latency). 


\section{Working Memory: Spatial Working Memory task (SWM)}

The SWM is a self-paced task during which an increasing number of boxes (four, six, and eight) is presented in four trials on the screen. Participants are supposed to find blue tokens which are hidden inside the boxes by touching the boxes and thereby opening them. In each trial the same number of tokens has to be found as the number of boxes presented on the screen. Participants are informed that once a token has been found within a particular box, the box will not be used again to hide a token. This tests measures executive functions (strategy score) as well as working memory errors (between and within errors). Between errors occur when a participant revisited a box in which a token had previously been found, whereas within errors are the number of times a participant revisited a box already found to be empty during the same search sequence. Strategy score describes the use of an efficient search strategy by beginning with a particular box and then returning to that box when a blue token was found to start the new search sequence. High strategy scores represent poor use of a strategy.

\section{Risk-based Decision-making: Cambridge Gambling Task (CGT)}

The CGT assesses decision-making and risk-taking behaviour outside a learning context. Participants start the task with 100 points. They are presented with 10 boxes on the screen. The boxes are either red or blue. The ration of red and blue boxes varies across trials (9:1, 8:2, 7:3, 6:4, 5:5). Participants have to decide whether a randomly hidden token is more likely to be in a red or blue box. Subsequently, participants bet on their decision by selecting a proportion of their points. The proportion of points (5\%, 25\%, 50\%, 75\%, 95\%) that can be selected are presented in either ascending or descending order. The outcome measures of this task are: the proportion of trials on which subjects chose the more likely outcome (quality of decision making) and the proportion of current points that the subject stakes on each gamble when the more likely outcome is selected (risk taking).

\section{Statistical analysis}

Data were analyzed using STATISTICA 8.0 for Windows (StatSoft). For 5D-ASC ratings, a repeated-measures ANOVA with drug (Pla, LSD, Ket+LSD) and subscale (experience of unity, spiritual experience, blissful state, insightfulness, disembodiment, impaired control and cognition, anxiety, complex imagery, elementary imagery, audio-visual synesthesiae, changed meaning of percepts) as within-subject factors were computed. For CANTAB outcome variables (IED: number of errors adjusted, numbers of errors, and total latency; SWM: between errors, within errors, and strategy; CGT: quality of decision making, risk taking) repeated-measures ANOVAs with drug (Pla, LSD, Ket+LSD) as within-subject 
factor was computed. For the IED stage (1-9) was introduced as additional within-subject factor. For the SWM, stage (4, 6, 8 boxes) was introduced as additional within-subject factor. For the CGT risk ratio (9:1, 8:2, 7:3, 6:4) was introduced as additional within-subject factors. Tukey post-hoc comparisons followed significant main effects or interactions. Pearson's correlation analyses between CANTAB change scores (drug minus placebo) and 5D-ASC change scores (drug minus placebo) and verbal IQ were computed in case of significant drug effects on CANTAB outcome measures. Statistical comparisons of all data were carried out on a significance level set at $\mathrm{p}<0.05$ (two-tailed). 


\section{Results}

\section{D-ASC}

There was a significant drug $x$ subscale interaction $(F(20,480)=15.10$, $\mathrm{p}<0.000001$ ) (Fig. 1), a significant main effect of subscale $(\mathrm{F}(10,240)=16.62$, $\mathrm{p}<0.000001)$ and a significant main effect of drug $(\mathrm{F}(2,48)=85.06, \mathrm{p}<0.000001)$. Tukey post-hoc tests revealed that LSD significantly increased all subscale scores compared to Pla and Ket+LSD (all $\mathrm{p}<0.0001$ ) except for anxiety ( $\mathrm{p}>0.7$ ). There were no significant differences between Pla and Ket+LSD in any subscale score (all p>0.9)

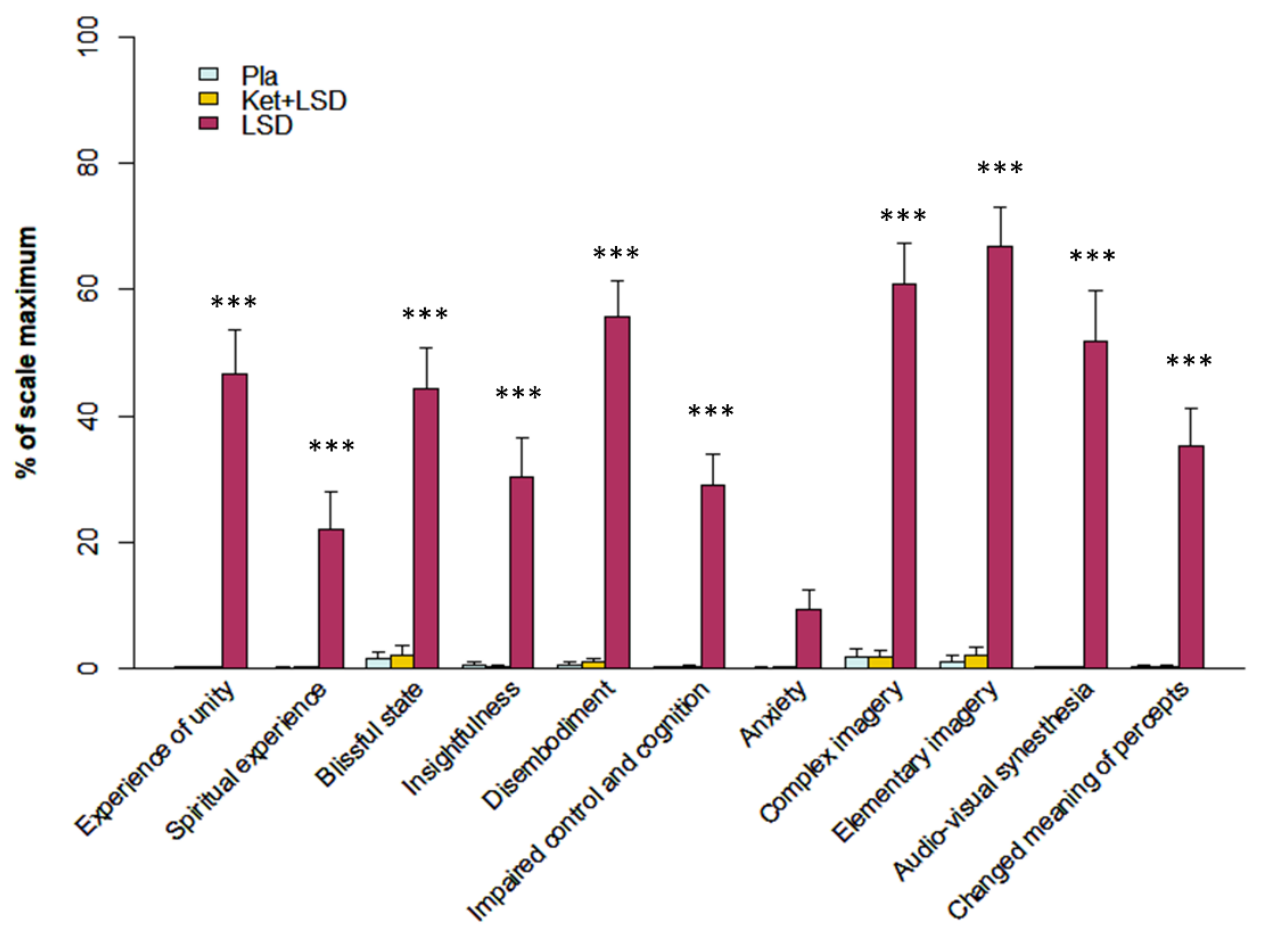

Figure 1: Subjective drug effects assessed with the 5D-ASC questionnaire $720 \mathrm{~min}$ after substance administration. LSD significantly increased all scale scores compared to Pla and Ket+LSD (all $\mathrm{p}<0.0001$ ), except for anxiety ( $\mathrm{p}>0.7$ ). Data are expressed as mean + SEM. ${ }^{* * *} \mathrm{p}<0.0001$, corrected.

\section{Intra/Extra-Dimensional shift task}

The stages completed did not differ between drug conditions $(F(2,48)=1.5, \mathrm{p}>0.2$; mean (SD) Pla: 8.84 (0.11), Ket+LSD: 8.76 (0.13), LSD: 8.68 (0.15)). However, for number of errors adjusted, there was a significant effect of $\operatorname{drug}(\mathrm{F}(2,48)=4.9, \mathrm{p}<0.05))$ with more errors in the LSD condition compared to Pla $(\mathrm{p}<0.05)$ and Ket+LSD $(\mathrm{p}<0.05)$ (Fig 2A). To further investigate at which stage the errors occurred, we computed a repeated-measures ANOVA with stage and drug as within-subject factors. We found a significant drug $\mathrm{x}$ stage interaction 
( $F(16,384=1.81, p<0.05)$ (Fig. 2B), a significant main effect of stage $(F(8,192)=9.10$, $\mathrm{p}<0.00001)$, and a significant main effect of drug $(\mathrm{F}(2,48)=4.50, \mathrm{p}<0.05)$. Tukey post-hoc tests revealed that LSD significantly increased the number of errors in stage 8 (EDS) compared to Pla $(\mathrm{p}<0.0001)$ and Ket+LSD $(\mathrm{p}<0.01)$, but not in any other stage compared to both, Pla and Ket+LSD (all p>0.9). There were no significant differences in the number of errors in any stage between Pla and Ket+LSD ( $>>0.9)$. For total latency, there was a significant drug $\mathrm{x}$ stage interaction $(\mathrm{F}(16,384)=1.94, \mathrm{p}<0.05) \quad$ (Fig. 2C), a significant main effect of stage $(F(8,192)=12.47, p<0.00001)$, and a significant main effect of drug $(F(2,48)=7.46, p<0.01)$. Tukey post-hoc tests revealed that LSD significantly increased latency in stage 8 (EDS) compared to Pla $(\mathrm{p}<0.0001)$ and Ket $+\operatorname{LSD}(\mathrm{p}<0.0001)$, but not in any other stage compared to Pla ( $>>0.6)$ or Ket+LSD ( $>0.7$ ). There were no significant differences in total latency scores in any stage between Pla and Ket+LSD (all p>0.9).
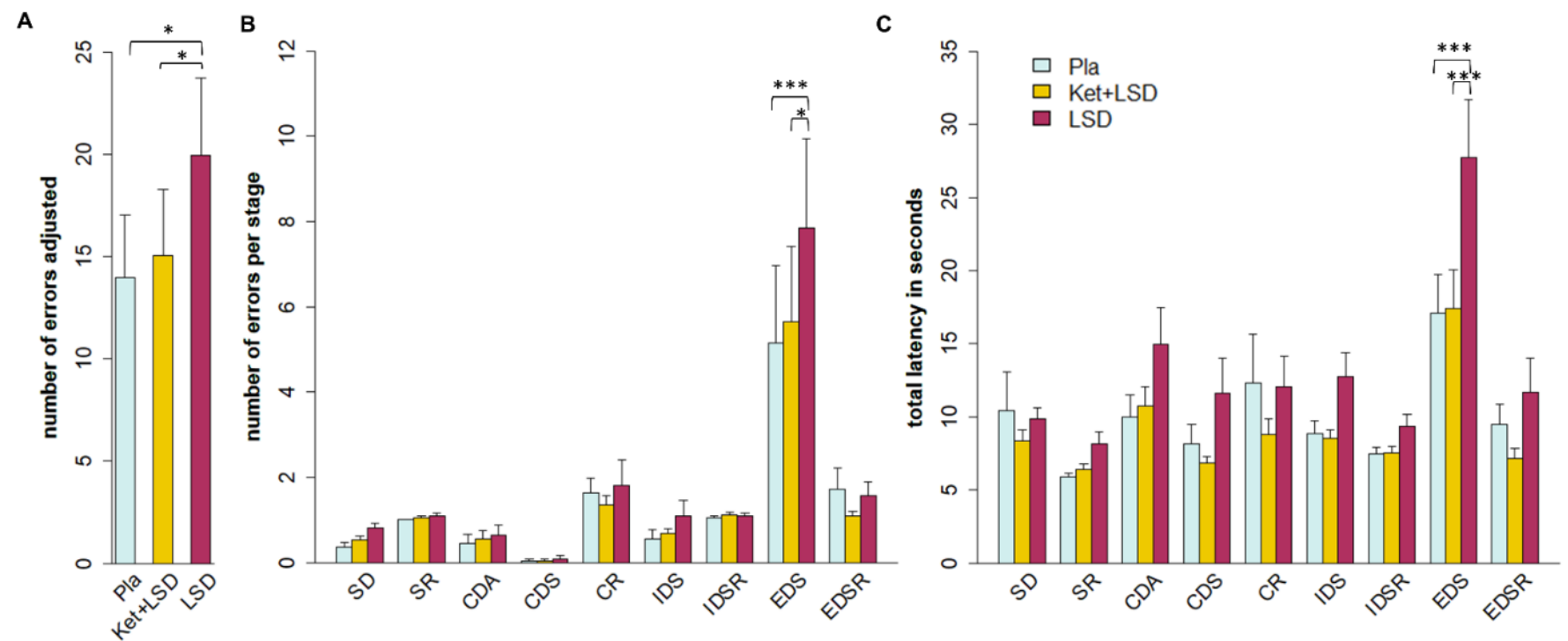

Figure 2: Intra/Extra-Dimensional shift task. A) displays the total number of errors adjusted for stages completed. LSD significantly increased the number of errors adjusted compared to Pla and Ket+LSD (both $\mathrm{p}<0.05$, corrected). B) displays the number of errors in each stage. LSD significantly increased the number of errors in the EDS stage compared to Pla $(\mathrm{p}<0.0001$, corrected) and Ket+LSD $(\mathrm{p}<0.01)$. C) displays the total latency (mean time to reach a decision within individual stages). LSD significantly increased latency in the EDS stage compared to Pla and Ket+LSD (both $\mathrm{p}<0.0001$ ). Data are expressed as mean + SEM. ${ }^{*} \mathrm{p}<0.01$, ${ }^{* * *} \mathrm{p}<0.0001$. IED stages: $S D$ simple discrimination, $S R$ simple reversal, $C D A$ compound discrimination adjacent, $C D S$ compound discrimination superimposed, $C R$ compound reversal, IDS intra-dimensional shift, IDSR intra-dimensional shift reversal, EDS extra-dimensional shift, EDSR extra-dimensional shift reversal 
Pokorny et al.

\section{Spatial Working Memory}

For between errors, there was a significant drug $x$ stage interaction $(F(4,96)=4.59$, $\mathrm{p}<0.01)$ (Fig. 3A), a significant main effect of drug $(\mathrm{F}(2,48)=7.14, \mathrm{p}<0.01)$, and a significant main effect of stage $(\mathrm{F}(2,48)=34.88, \mathrm{p}<0.00001)$. Tukey post-hoc tests revealed that participants made significantly more between errors in the LSD condition than in the Pla condition when six boxes were presented $(\mathrm{p}<0.01)$. Further, LSD significantly increased between errors when eight boxes were presented compared to both Pla $(\mathrm{p}<0.001)$ and Ket $+\mathrm{LSD}$ $(\mathrm{p}<0.001)$. There were no significant differences in the number of between errors between Pla and Ket+LSD at any stage (all $\mathrm{p}>0.6$ ). For within errors, there was no significant drug $\mathrm{x}$ stage interaction $(F(4,96)=1.00, \mathrm{p}>0.4)$ or main effect of drug $(\mathrm{F}(2,48)=1.84, \mathrm{p}>0.1)$ (Fig. 3B). However, we found a significant main effect of stage $(F(2,48)=5.09, p<0.01)$. For the strategy score, there was a significant drug $x$ stage interaction $(F(4,96)=4.11, p<0.01)$ (Fig. 3C), a significant main effect of stage $(F(2,48)=205.50, p<0.00001)$, but no significant main effect of drug $(F(2,48)=1.48, p>0.2)$. Tukey post-hoc tests revealed that the strategy score was increased (reflecting poor use of a strategy) under LSD compared to Pla and Ket+LSD when eight boxes were presented (both $\mathrm{p}<0.01$ ). There was no significant difference in the strategy scores between Pla and Ket+LSD at any stage (all p>0.9).

A

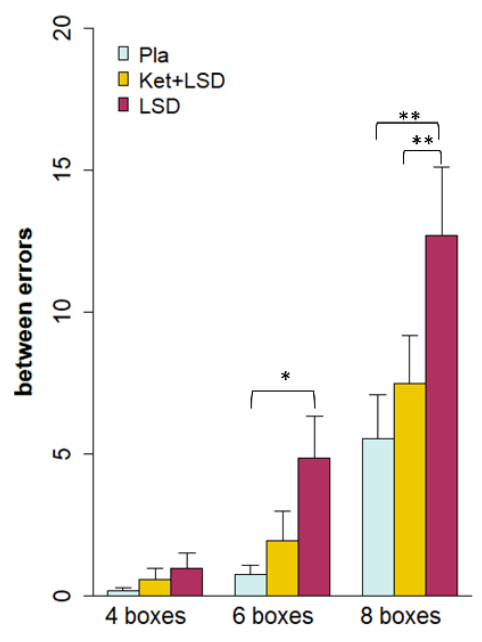

B

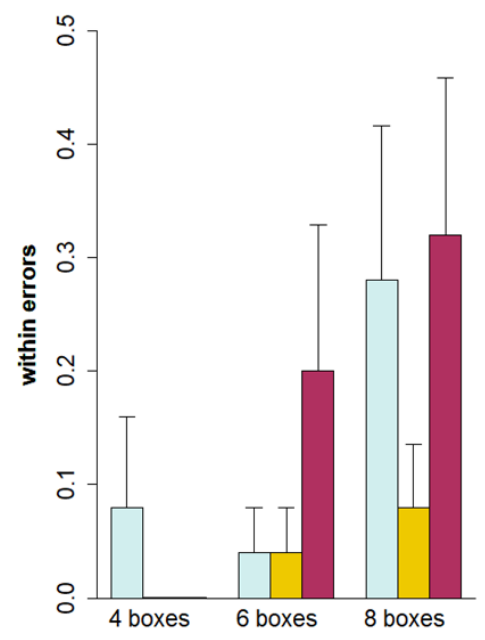

C

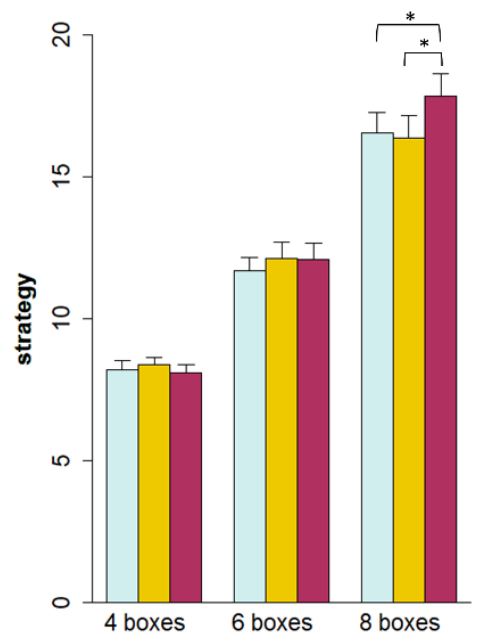

Figure 3: Spatial Working Memory. A) displays between errors. LSD significantly increased the number of between errors when six boxes were presented compared to Pla ( $<<0.01$, corrected). When eight boxes were presented, LSD significantly increased between errors compared to Pla and Ket+LSD (both $\mathrm{p}<0.001$, corrected). B) displays within errors. There were no significant drug effects for number of within errors. C) displays the strategy scores. LSD reduced the use of an efficient search strategy when eight boxes were presented compared to Pla and Ket+LSD (both $\mathrm{p}<0.01$ ). Data are expressed as mean + SEM. ${ }^{*} \mathrm{p}<0.01$, ${ }^{* *} \mathrm{p}<0.001$. 


\section{Cambridge Gambling Task}

For quality of decision making, there was no significant drug $\mathrm{x}$ risk ratio $(\mathrm{F}(6,144)=0.38$, $\mathrm{p}>0.8$ ), interaction (Fig. 4A), and no main effects of drug $(\mathrm{F}(2,48)=0.50, \mathrm{p}>0.6)$ or risk ratio $(\mathrm{F}(3,72)=1.93, \mathrm{p}>0.1)$. For risk taking (Fig. 4B), there was no significant drug $\mathrm{x}$ risk ratio $(F(6,144)=1.04, p>0.4)$ interaction, and no significant main effect for drug $F(2,48)=1.05$, $\mathrm{p}>0.3)$. However, there was a significant main effect of risk ratio $(F(3,72)=108.06, p<0.000001)$ for risk taking, indicating that participants made higher bets when the risk ratio was lower.

A

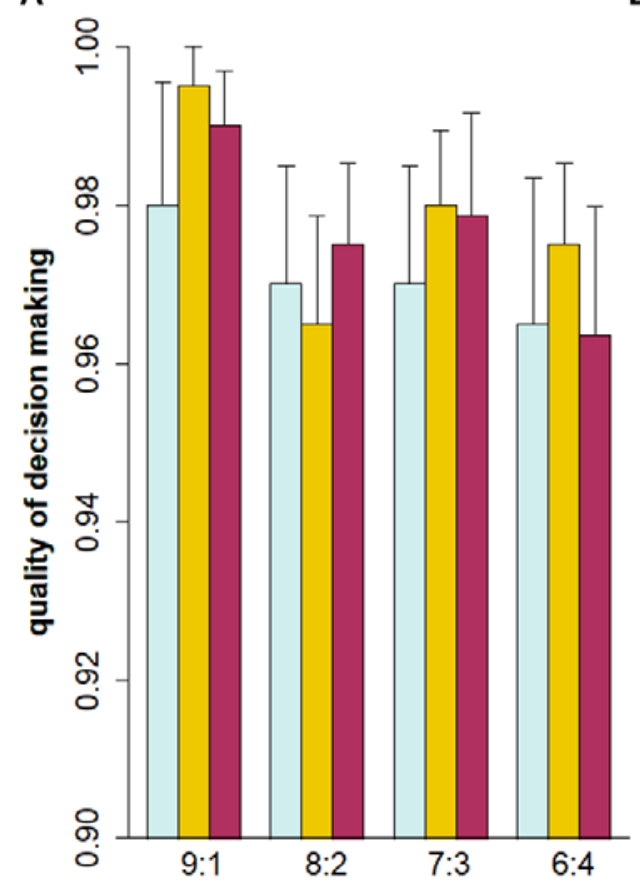

B

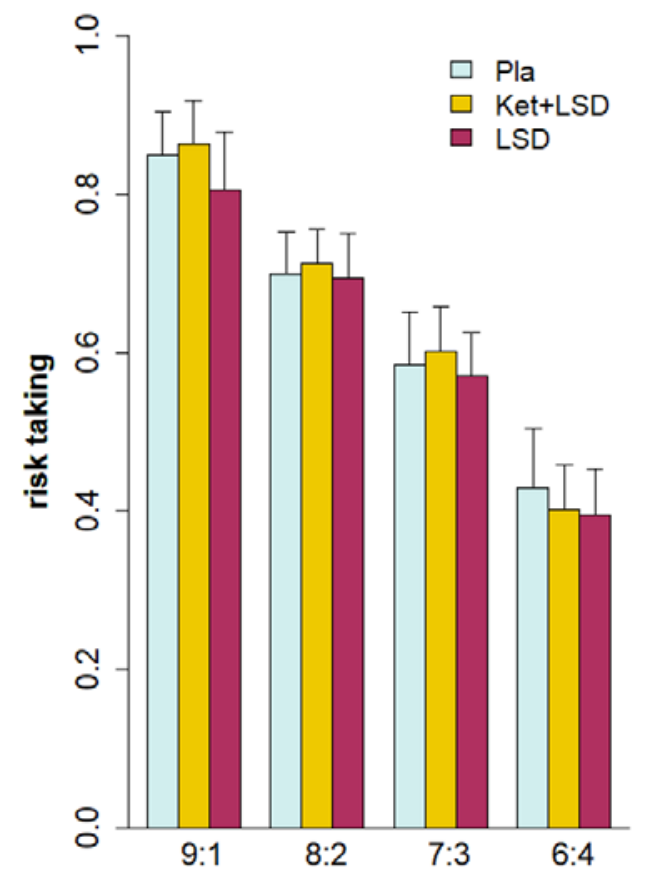

Figure 4: Cambridge Gambling Task. No significant effects of drug were found for A) quality of decision-making or B) risk taking. Data are expressed as mean + SEM.

\section{Correlations}

After Bonferroni correction for multiple comparisons there were no significant correlations between change scores of CANTAB outcome measures and change scores of 5DASC subscale scores (all p>0.1, corrected). Furthermore, IQ was not correlated with change scores of CANTAB outcome measures (all p>0.3, corrected). 
Pokorny et al.

\section{Discussion}

Trans-diagnostic deficits in cognitive abilities are highly prevalent in psychiatric and neurological disorders, but are insufficiently improved by current treatment approaches (Millan, et al, 2012). The current study closes major knowledge gaps in the field via the administration of LSD together with a 5- $\mathrm{HT}_{2 \mathrm{~A}}$ receptor antagonist and the application of standardized and computerized cognitive tasks that capture the most relevant cognitive domains impaired in psychiatric disorders (Jessen, et al, 2018). We show that I) acutely administered LSD significantly impaired executive functions and working memory compared to placebo, II) risk-based decision-making was unaffected by LSD, and III) LSD-induced cognitive deficits and subjective symptoms were dependent on 5- $\mathrm{HT}_{2 \mathrm{~A}}$ receptor stimulation.

\section{LSD impairs cognitive flexibility and executive functions on the Intra/Extra-Dimensional shift task}

LSD led to a significant increase in error rates and increased latency in the EDS stage of the IED task compared to placebo. These impairments were normalized in the Ket+LSD condition. Impairments in the EDS stage are interpreted as signs of preservation of a previously established attentional set (Elliott, et al, 1995). Extradimensional shifting requires being able to inhibit the previously established attentional set and shift attention between stimulus dimensions. Therefore, impairments in EDS are interpreted as reduced cognitive flexibility (Chamberlain, et al, 2007). Processes that may underlie this LSD-induced decrease in cognitive flexibility are deficits in executive functions, in particular increased susceptibility to distraction from task-irrelevant stimuli (Jazbec, et al, 2007). This interpretation is supported by previous studies in healthy humans which have shown that psilocybin, a structurally related serotonergic hallucinogen and 5- $\mathrm{HT}_{2 \mathrm{~A}}$ receptor agonist, led to a significant decline of correct detection and an increase of false alarm in the AX continuous performance test (Umbricht, et al, 2003) and to a significant reduction in attentional tracking ability on a multiple-object tracking task, an effect attenuated by ketanserin (Carter, et al, 2007). The authors of the later study suggest that the impaired attentional performance under psilocybin may reflect a reduced ability to suppress or ignore distracting stimuli rather than reduced attentional capacity per se. This interpretation may also explain seemingly contradictory findings reporting increases in psychological flexibility under the influence of other psychedelic substances (Kuypers, et al, 2016) . Here, participants were instructed to provide alternative interpretations of the presented stimulus material. However, the applied tasks did not require the suppression of distracting elements which may explain the differing results. In contrast to our current finding, an early study with 
LSD found no significant change in performance on the Wisconsin Card Sorting Test (Primac, et al, 1957), of which the IED is the computerised analogue. It seems likely that this previous study was not able to detect LSD-induced effects due to the small sample size $(n=10)$. Importantly, LSD-induced impairments in cognitive flexibility and executive functions were normalized by ketanserin, therefore pinpointing the crucial role of the 5- $\mathrm{HT}_{2 \mathrm{~A}}$ receptor in these cognitive functions.

\section{LSD impairs executive functions and working memory on the Spatial Working Memory task}

LSD compared to placebo led to a significant increase of between errors and decreased use of strategy in the SWM. An increase in between errors represents a deficit in working memory, since participants revisited boxes even though a token had already been found within the box. Deficits in strategy represent impairments in executive functions. Importantly, these effects were only present when cognitive load was high. These results are in line with previous studies investigating the effects of other psychedelic 5- $\mathrm{HT}_{2 \mathrm{~A}}$ agonists on spatial working memory. Psilocybin has been shown to dose-dependently impair performance on a spatial working memory task (Wittmann, et al, 2007). Furthermore, ayahuasca acutely impaired working memory (Bouso, et al, 2013). Similarly to results obtained on the IED, pre-treatment with ketanserin normalized LSD-induced working memory and executive function deficits on the SWM in the current study.

\section{The role of the $5-\mathrm{HT}_{2 A}$ receptor in $\mathrm{LSD}$-induced cognitive impairments}

On both, the IED and the SWM task, blocking the 5-HT2A receptor with ketanserin prevented LSD-induced cognitive deficits, therefore pointing to the importance of this receptor system in working memory and executive functions. This result is in line with a recent computational study indicating that $5-\mathrm{HT}_{2 \mathrm{~A}}$ receptors contribute to spatial working memory tasks (Cano-Colino, et al, 2014). The hippocampus is particularly involved in spatial memory and has moderate to high levels of 5- $\mathrm{HT}_{2 \mathrm{~A}}$ receptors (Dwivedi and Pandey, 1998). Injections of the 5- $\mathrm{HT}_{2 \mathrm{~A}}$ receptor antagonist ritanserin in rat hippocampus significantly improved spatial memory in the Morris Water Maze task (Naghdi and Harooni, 2005). Furthermore, the prefrontal cortex (PFC) is implicated in higher-order executive tasks such as working memory, attention, and executive function (Millan, et al, 2012). The PFC is linked to the parietal cortex, which exerts a modulatory influence on attention and working memory (Millan, et al, 2012). Both structures have a particular high density of 5-HT2A receptors, and they exert top-down modulatory influence on subcortical regions including the hippocampus (Pompeiano, et al, 1994). Clinical evidence corroborates the current results pinpointing the importance of the 5- 
$\mathrm{HT}_{2 \mathrm{~A}}$ receptor in cognitive abilities: Atypical antipsychotics, which have 5- $\mathrm{HT}_{2 \mathrm{~A}}$ receptor antagonistic properties, have been shown to be advantageous for treating cognitive impairments in schizophrenia compared to classic antipsychotics (Meltzer, et al, 2012). There are qualitative similarities between hallucinogen-induced alterations in information processing and the symptoms of an early phase of schizophrenic psychoses (Geyer and Vollenweider, 2008). Specifically, sensorimotor gating as indexed by prepulse inhibition (PPI) is impaired in patients with schizophrenia and related to cognitive deficits (Braff, et al, 2001). LSD and psilocybin disrupt PPI in healthy subjects, an effect that was also correlated with impairments of sustained attention in healthy humans (Gouzoulis-Mayfrank, et al, 1998; Quednow, et al, 2012; Schmid, et al, 2015; Vollenweider, et al, 2007). Importantly, it has been shown that the disruptions of PPI induced by psilocybin in humans and LSD in rats are reversed by $5-\mathrm{HT}_{2 \mathrm{~A}}$ receptor antagonists (Halberstadt and Geyer, 2010; Ouagazzal, et al, 2001; Quednow, et al, 2012). Together these results suggest that the $5-\mathrm{HT}_{2 \mathrm{~A}}$ receptor system may be a promising target in the treatment of trans-diagnostic impairments in working memory and executive functions.

\section{LSD does not affect risk-based decision making on the Cambridge GamblingTask}

Interestingly, in the CGT, no significant differences between the three drug conditions were found. LSD did not influence the quality of decision making and risk taking. Decision making and risk taking may therefore represent cognitive domains that are not modulated by $5-\mathrm{HT}_{2 \mathrm{~A}}$ receptor signalling. This is in line with a recent study that showed that psilocybin had no effect on moral decision-making (Pokorny et al., 2017). Furthermore, this result supports previous reports that associate risk-based decision-making behaviour with dopaminergic signalling, whereas the serotonin system has been suggested to play a role in the regulation of cognitive biases, and therefore the appraisal of reinforcers when selecting between actions, in particular in a learning context (Rogers, 2011). The CGT, however, is not relying on learning, and riskbased decision making on the CGT may therefore not be sensitive to alterations in $5-\mathrm{HT}_{2 \mathrm{~A}}$ receptor signalling. Yet, LSD also has affinity for dopamine receptors and animal studies have reported a first 5- $\mathrm{HT}_{2 \mathrm{~A}}$ receptor mediated and a second D2 receptor mediated phase of action (Marona-Lewicka and Nichols, 2007; Marona-Lewicka, et al, 2005). However, the involvement of the dopaminergic system in this study is unlikely as pretreament with ketanserin normalized the LSD-induced effects not only in the CANTAB tasks 220 minutes after drug intake but also the retrospective rated psychological effects measured with the 5D-ASC. Therefore, the present results indicate that the effects of LSD in humans are primarily mediated via $5-\mathrm{HT}_{2 \mathrm{~A}}$ receptor activation. This is in line with recent reports, that LSD in humans increased the levels of prolactin and cortisol, which are markers for serotonergic drug activity (Schmid, et al, 2015). 
However, it is possible that higher doses of LSD are needed to modulate dopaminergic activity and potentially induce alterations in risk-based decision making.

\section{Correlations and limitations}

Cognitive impairments in the IED and SWM did not correlate with the LSD-induced subjective effects. Importantly, this suggests that cognitive processes under LSD are not confounded by psychedelic effects, in particular visual inaccuracies or disturbances. Furthermore, LSD-induced impairments were not related to individuals’ IQ, suggesting that 5$\mathrm{HT}_{2 \mathrm{~A}}$ receptor stimulation by LSD impaired working memory and executive functions independently of general intelligence.

Previous studies have shown that the selective 5- $\mathrm{HT}_{2 \mathrm{~A}}$ receptor antagonist ketanserin is suitable for studying the role of $5-\mathrm{HT}_{2 \mathrm{~A}}$ receptors in human performance and to investigate the specific contribution of the 5-HT2A receptor to effects of psychedelic drugs (Carter, et al, 2007; Kometer, et al, 2012; Liechti, et al, 2000; Quednow, et al, 2012; Vollenweider, et al, 1998; Preller, et al, 2017). A limitation of the present study is the lack of a fourth drug condition investigating the effect of ketanserin alone. However, previous studies have shown that ketanserin neither led to any significant differences in subjective drug effects assessed with the 5D-ASC (Carter, et al, 2007; Kometer, et al, 2012), nor to performance changes in cognitive tasks such as a spatial working memory task (Carter, et al, 2007), or the Stroop task (Quednow, et al, 2012).

\section{Conclusion}

In conclusion, the present study pinpoints the role of the 5- $\mathrm{HT}_{2 \mathrm{~A}}$ receptor in cognitive processes, in particular executive functions, cognitive flexibility, and spatial working memory. However, risk-based decision-making outside a learning context was unaffected by LSD and is therefore potentially not mediated by the $5-\mathrm{HT}_{2 \mathrm{~A}}$ receptor. Blocking the 5-HT2A receptor by ketanserin normalized both LSD-induced cognitive impairments and subjective drug effects. As altered 5-HT $2 \mathrm{~A}$ receptor density and cognitive dysfunctions are found in several psychiatric disorders such as in schizophrenia, autism, and obsessive compulsive disorder (Millan, et al, 2012), this receptor subtype represents a promising target to help understanding the neurophramacological basis of cognitive processes and to improve treatment in affected patients. 
Pokorny et al.

\section{Funding and Disclosure}

Funding for this study was provided by Swiss Neuromatrix Foundation (grant number 2015-2056) and Heffter Research Institute (grant number 1-190413); both foundations had no further role in study design; in the collection, analysis and interpretation of data; in the writing of the report; and in the decision to submit the paper for publication. All authors declare no conflict of interest.

\section{Acknowledgments}

We thank Amanda Planzer, Jan Flemming, and Rainer Krähenmann for their assistance in data collection. 


\section{References}

Bouso JC, Fabregas JM, Antonijoan RM, Rodriguez-Fornells A, Riba J (2013). Acute effects of ayahuasca on neuropsychological performance: differences in executive function between experienced and occasional users. Psychopharmacology 230(3): 415-424.

Braff DL, Geyer MA, Swerdlow NR (2001). Human studies of prepulse inhibition of startle: normal subjects, patient groups, and pharmacological studies. Psychopharmacology 156(2-3): 234-258.

Cano-Colino M, Almeida R, Gomez-Cabrero D, Artigas F, Compte A (2014). Serotonin regulates performance nonmonotonically in a spatial working memory network. Cerebral cortex (New York, N.Y. 1991) 24(9): 2449-2463.

Carhart-Harris RL, Kaelen M, Bolstridge M, Williams TM, Williams LT, Underwood R, et al (2016). The paradoxical psychological effects of lysergic acid diethylamide (LSD). Psychological medicine 46(7): 1379-1390.

Carter OL, Hasler F, Pettigrew JD, Wallis GM, Liu GB, Vollenweider FX (2007). Psilocybin links binocular rivalry switch rate to attention and subjective arousal levels in humans. Psychopharmacology 195(3): 415-424.

Chamberlain SR, Fineberg NA, Menzies LA, Blackwell AD, Bullmore ET, Robbins TW, et al (2007). Impaired cognitive flexibility and motor inhibition in unaffected first-degree relatives of patients with obsessive-compulsive disorder. The American journal of psychiatry 164(2): 335-338.

Claesdotter E, Cervin M, Åkerlund S, Råstam M, Lindvall M (2018). The effects of ADHD on cognitive performance. Nordic journal of psychiatry 72(3): 158-163.

Désaméricq G, Schurhoff F, Meary A, Szöke A, Macquin-Mavier I, Bachoud-Lévi AC, et al (2014). Long-term neurocognitive effects of antipsychotics in schizophrenia: a network meta-analysis. European journal of clinical pharmacology 70(2): 127-134.

Dittrich A (1998). The standardized psychometric assessment of altered states of consciousness (ASCs) in humans. Pharmacopsychiatry 31 Suppl 2: 80-84.

Dwivedi Y, Pandey GN (1998). Quantitation of 5HT2A receptor mRNA in human postmortem brain using competitive RT-PCR. Neuroreport 9(17): 3761-3765.

Elliott R, McKenna PJ, Robbins TW, Sahakian BJ (1995). Neuropsychological evidence for frontostriatal dysfunction in schizophrenia. Psychological medicine 25(3): 619-630.

Geyer MA, Vollenweider FX (2008). Serotonin research: contributions to understanding psychoses. Trends in pharmacological sciences 29(9): 445-453.

Gouzoulis-Mayfrank E, Heekeren K, Thelen B, Lindenblatt H, Kovar KA, Sass H, et al (1998). Effects of the hallucinogen psilocybin on habituation and prepulse inhibition of the startle reflex in humans. Behavioural pharmacology 9(7): 561-566.

Halberstadt AL, Geyer MA (2010). LSD but not lisuride disrupts prepulse inhibition in rats by activating the 5-HT(2A) receptor. Psychopharmacology 208(2): 179-189.

Hill SK, Bishop JR, Palumbo D, Sweeney JA (2010). Effect of second-generation antipsychotics on cognition: current issues and future challenges. Expert review of neurotherapeutics 10(1): 43-57.

Jagust W (2018). Imaging the evolution and pathophysiology of Alzheimer disease. Nature reviews. Neuroscience 19(11): 687-700.

Jazbec S, Pantelis C, Robbins T, Weickert T, Weinberger DR, Goldberg TE (2007). Intradimensional/extra-dimensional set-shifting performance in schizophrenia: impact of distractors. Schizophrenia research 89(1-3): 339-349.

Jessen K, Mandl RCW, Fagerlund B, Bojesen KB, Raghava JM, Obaid HG, et al (2018). Patterns of Cortical Structures and Cognition in Antipsychotic-Naïve Patients With First-Episode Schizophrenia: A Partial Least Squares Correlation Analysis. Biological psychiatry. Cognitive neuroscience and neuroimaging. 
Kometer M, Schmidt A, Bachmann R, Studerus E, Seifritz E, Vollenweider FX (2012). Psilocybin biases facial recognition, goal-directed behavior, and mood state toward positive relative to negative emotions through different serotonergic subreceptors. Biological psychiatry 72(11): 898_ 906.

Kraehenmann R, Pokorny D, Aicher H, Preller KH, Pokorny T, Bosch OG, et al (2017). LSD Increases Primary Process Thinking via Serotonin 2A Receptor Activation. Frontiers in pharmacology 8: 814.

Kuypers KPC, Riba J, La Fuente Revenga M de, Barker S, Theunissen EL, Ramaekers JG (2016). Ayahuasca enhances creative divergent thinking while decreasing conventional convergent thinking. Psychopharmacology 233(18): 3395-3403.

Lehrl S (2005): Manual zum MWT-B, 5. Aufl. Spitta-Verl.: Balingen.

Liechti ME, Saur MR, Gamma A, Hell D, Vollenweider FX (2000). Psychological and physiological effects of MDMA ("Ecstasy") after pretreatment with the 5-HT(2) antagonist ketanserin in healthy humans. Neuropsychopharmacology official publication of the American College of Neuropsychopharmacology 23(4): 396-404.

Marona-Lewicka D, Nichols DE (2007). Further evidence that the delayed temporal dopaminergic effects of LSD are mediated by a mechanism different than the first temporal phase of action. Pharmacology, biochemistry, and behavior 87(4): 453-461.

Marona-Lewicka D, Thisted RA, Nichols DE (2005). Distinct temporal phases in the behavioral pharmacology of LSD: dopamine D2 receptor-mediated effects in the rat and implications for psychosis. Psychopharmacology 180(3): 427-435.

Meltzer HY, Massey BW, Horiguchi M (2012). Serotonin receptors as targets for drugs useful to treat psychosis and cognitive impairment in schizophrenia. Current pharmaceutical biotechnology 13(8): 1572-1586.

Mestre TA, Zurowski M, Fox SH (2013). 5-Hydroxytryptamine 2A receptor antagonists as potential treatment for psychiatric disorders. Expert opinion on investigational drugs 22(4): 411-421.

Millan MJ, Agid Y, Brune M, Bullmore ET, Carter CS, Clayton NS, et al (2012). Cognitive dysfunction in psychiatric disorders: characteristics, causes and the quest for improved therapy. Nature reviews. Drug discovery 11(2): 141-168.

Naghdi N, Harooni HE (2005). The effect of intrahippocampal injections of ritanserin (5HT2A/2C antagonist) and granisetron (5HT3 antagonist) on learning as assessed in the spatial version of the water maze. Behavioural brain research 157(2): 205-210.

Ouagazzal A, Grottick AJ, Moreau J, Higgins GA (2001). Effect of LSD on prepulse inhibition and spontaneous behavior in the rat. A pharmacological analysis and comparison between two rat strains. Neuropsychopharmacology official publication of the American College of Neuropsychopharmacology 25(4): 565-575.

Pantelis C, Wood SJ, Proffitt TM, Testa R, Mahony K, Brewer WJ, et al (2009). Attentional setshifting ability in first-episode and established schizophrenia: Relationship to working memory. Schizophrenia research 112(1-3): 104-113.

Pompeiano M, Palacios JM, Mengod G (1994). Distribution of the serotonin 5-HT2 receptor family mRNAs: comparison between 5-HT2A and 5-HT2C receptors. Brain research. Molecular brain research 23(1-2): 163-178.

Preller KH, Burt JB, Ji JL, Schleifer CH, Adkinson BD, Stämpfli P, et al (2018a). Changes in global and thalamic brain connectivity in LSD-induced altered states of consciousness are attributable to the 5-HT2A receptor. eLife 7.

Preller KH, Herdener M, Pokorny T, Planzer A, Kraehenmann R, Stämpfli P, et al (2017). The Fabric of Meaning and Subjective Effects in LSD-Induced States Depend on Serotonin 2A Receptor Activation. Current biology CB 27(3): 451-457. 
Preller KH, Schilbach L, Pokorny T, Flemming J, Seifritz E, Vollenweider FX (2018b). Role of the 5HT2Areceptor in self- and other-initiated social interaction in LSD-induced states - a pharmacological fMRI study. The Journal of neuroscience the official journal of the Society for Neuroscience.

Primac DW, Mirsky AF, Rosvold HE (1957). Effects of centrally acting drugs on two tests of brain damage. A.M.A. archives of neurology and psychiatry 77(3): 328-332.

Quednow BB, Kometer M, Geyer MA, Vollenweider FX (2012). Psilocybin-induced deficits in automatic and controlled inhibition are attenuated by ketanserin in healthy human volunteers. Neuropsychopharmacology official publication of the American College of Neuropsychopharmacology 37(3): 630-640.

Rock PL, Roiser JP, Riedel WJ, Blackwell AD (2014). Cognitive impairment in depression: a systematic review and meta-analysis. Psychological medicine 44(10): 2029-2040.

Rogers RD (2011). The roles of dopamine and serotonin in decision making: evidence from pharmacological experiments in humans. Neuropsychopharmacology official publication of the American College of Neuropsychopharmacology 36(1): 114-132.

Schmid Y, Enzler F, Gasser P, Grouzmann E, Preller KH, Vollenweider FX, et al (2015). Acute Effects of Lysergic Acid Diethylamide in Healthy Subjects. Biological psychiatry 78(8): 544-553.

Sheehan DV, Lecrubier Y, Sheehan KH, Amorim P, Janavs J, Weiller E, et al (1998). The MiniInternational Neuropsychiatric Interview (M.I.N.I.): the development and validation of a structured diagnostic psychiatric interview for DSM-IV and ICD-10. J Clin Psychiatry 59 Suppl 20: 22.

Studerus E, Gamma A, Vollenweider FX (2010). Psychometric evaluation of the altered states of consciousness rating scale (OAV). PloS one 5(8): e12412.

Umbricht D, Vollenweider FX, Schmid L, Grubel C, Skrabo A, Huber T, et al (2003). Effects of the 5HT2A agonist psilocybin on mismatch negativity generation and AX-continuous performance task: implications for the neuropharmacology of cognitive deficits in schizophrenia. Neuropsychopharmacology official publication of the American College of Neuropsychopharmacology 28(1): 170-181.

Vollenweider FX, Csomor PA, Knappe B, Geyer MA, Quednow BB (2007). The effects of the preferential 5-HT2A agonist psilocybin on prepulse inhibition of startle in healthy human volunteers depend on interstimulus interval. Neuropsychopharmacology official publication of the American College of Neuropsychopharmacology 32(9): 1876-1887.

Vollenweider FX, Vollenweider-Scherpenhuyzen MF, Babler A, Vogel H, Hell D (1998). Psilocybin induces schizophrenia-like psychosis in humans via a serotonin-2 agonist action. Neuroreport 9(17): 3897-3902.

Wang AM, Pradhan S, Coughlin JM, Trivedi A, DuBois SL, Crawford JL, et al (2019). Assessing Brain Metabolism With 7-T Proton Magnetic Resonance Spectroscopy in Patients With FirstEpisode Psychosis. JAMA psychiatry.

Wittmann M, Carter O, Hasler F, Cahn BR, Grimberg U, Spring P, et al (2007). Effects of psilocybin on time perception and temporal control of behaviour in humans. Journal of psychopharmacology (Oxford, England) 21(1): 50-64.

Wunderli MD, Vonmoos M, Niedecker SM, Hulka LM, Preller KH, Baumgartner MR, et al (2016). Cognitive and emotional impairments in adults with attention-deficit/hyperactivity disorder and cocaine use. Drug and alcohol dependence 163: 92-99.

Zhang G, Stackman RW (2015). The role of serotonin 5-HT2A receptors in memory and cognition. Frontiers in pharmacology 6: 225. 